DOI: 10.2478/romneu-2014-0003

\title{
Radio-induced brain lesions
}

\section{Mircea Radu Gorgan ${ }^{1}$, Andrei Giovani ${ }^{2}$, Aurelia Mihaela Sandu ${ }^{2}$, Felix Mircea Brehar ${ }^{1}$, Narcisa Bucur ${ }^{2}$, Angela Neaç̧u ${ }^{2}$, Catioara Fănica Cristescu ${ }^{3}$}

${ }^{1}$ Clinic of Neurosurgery, Fourth Department of Neurosurgery, Emergency Clinical Hospital Bagdasar-Arseni, University of Medicine and Pharmacy "Carol Davila" Bucharest

${ }^{2}$ Clinic of Neurosurgery, Fourth Department of Neurosurgery, Emergency Clinical Hospital Bagdasar-Arseni, Bucharest

${ }^{3}$ Department of Anesthesiology and Intensive Care, Emergency Clinical Hospital BagdasarArseni, Bucharest

\section{Abstract}

Introduction: Radiotherapy, an important tool in multimodal oncologic treatment, can cause radio-induced brain lesion development after a long period of time following irradiation.

Material and method: We report 4 cases with radio-induced brain lesions, admitted into the Fourth Department of Neurosurgery, Emergency Clinical Hospital Bagdasar-Arseni, during a 4 years period of time.

Results: Two patients had meningiomas and two had unruptured cavernomas. Other side effects of radiotherapy, such as diffuse brain atrophy, leukoencephalopathy, optic atrophy, panhypopituitarism were also noted. The two patients with large meningiomas underwent surgery, with good outcome. Observation was the choice for the two asymptomatic cavernomas. Panhypopituitarism needed synthetic hormonal replacement therapy.

Conclusions: Radiotherapy can cause long-term complications and can induce development of new brain lesions into previous radiation area. Meningiomas and cavernomas can be radio-induced brain lesions. Meningiomas can grow to large size, requiring surgery. Unruptured asymptomatic cavernomas can be left in place and patients are followed clinical and with serial imaging. Other findings after radiotherapy are diffuse brain atrophy, leukoencephalopathy, optic atrophy and panhypopituitarism.

Key words: cavernoma, meningioma, radio-induced lesions, radiotherapy.

\section{Introduction}

Radiotherapy is an important tool in oncology. It is widely used in multimodal treatment of malignant brain tumors. Radiotherapy is not side effects free. Consequences can occur immediately after irradiation or after a period of time. Side 
effects of radiotherapy include brain radionecrosis, malignant transformation of benign tumors and onset of new distinctive tumors.(9)

In 1902 Frieben first described the etiologic role of radiation in tumor induction and Lacassagne confirmed this theory on experimental animals in 1933.(4)

Radio-induced brain lesions occur inside a previously radiation area. They are not present at the time of radiotherapy and occur after a sufficiently long period of time following radiotherapy. Lesions must have a different histopathological diagnosis compare with the primary tumor and patients lack genetic predisposition for second tumor occurrence.(2;7;11) Most common radio-induced brain lesions are: meningiomas, vestibular schwannomas, gliomas, cavernomas, gliomas, sarcomas, etc. $(10 ; 11)$

\section{Material and Method}

We report 4 cases with radio-induced brain lesions, admitted into the Fourth Department of Neurosurgery, Emergency Clinical Hospital Bagdasar-Arseni, during a 4 years period of time, from 2010 to 2013. We retrospectively reviewed medical records, analyzing: demographic data, history, previous treatment, signs and symptoms at admission, general exam, neurological exam, cerebral imaging, decision making, treatment and prognosis.

\section{Case 1}

BF, M, 39 years old, was admitted in 2001 in our department and was diagnosed with third ventricle tumor with upward extension into the both lateral ventricles. The patients underwent surgery, we performed a transcortical approach and total tumor resection. Histopathological exam was anaplastic ependymoma. Postoperative the patient underwent whole brain radiotherapy and spinal radiotherapy.

Thirteen years later, in 2013, the patient was admitted with headache and gait disturbances. Cerebral MRI showed no tumor recurrence within the ventricular system and left parasagittal parietooccipital tumor, with dural attachment, well-defined, strongly enhancing contrast, sizing 38/37/38 mm (figure 1).

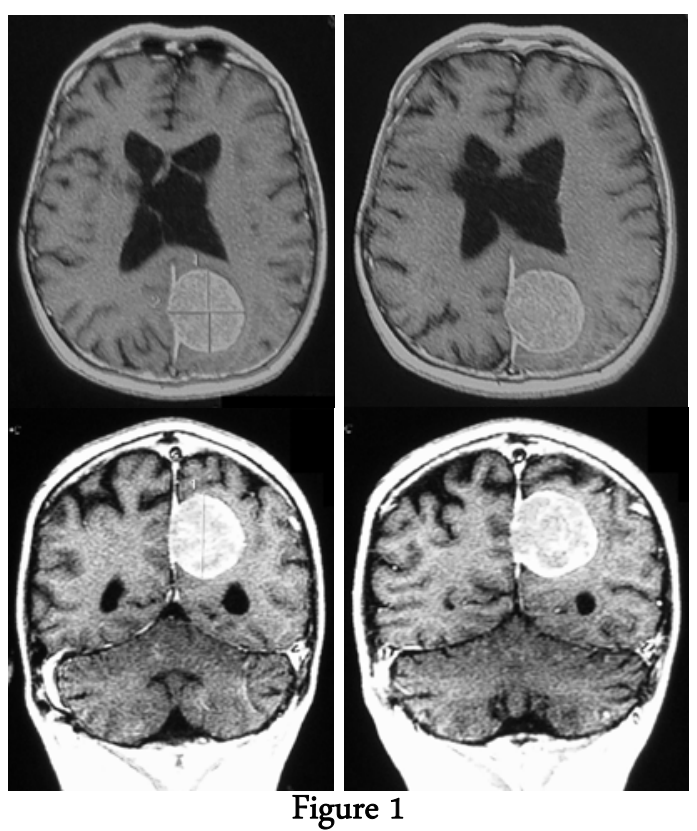

Cerebral MRI. No tumor recurrence within the ventricular system. Left parasagittal PO tumor, with dural attachment, well-defined, strongly enhancing contrast, sizing 38/37/38 mm.

The patient was diagnosed with radioinduced left parasagittal parieto-occipital 
tumor and panhypopituitarism. The patient underwent second surgery and we performed total tumor resection. Histopathological exam was transitional meningioma. Postoperative outcome was favorable, with no neurological deficits.

\section{Case 2}

BML, M, 41 years old, was admitted for the first time, in 1992 with left lateral ventricle tumor and secondary hydrocephalus. The patient underwent surgery, we performed a transcortical approach and total tumor resection. Histopathological exam was germinoma. Latter the patient needed ventriculoperitoneal shunt for secondary hydrocephalus. Postoperative the patient underwent whole brain radiotherapy.

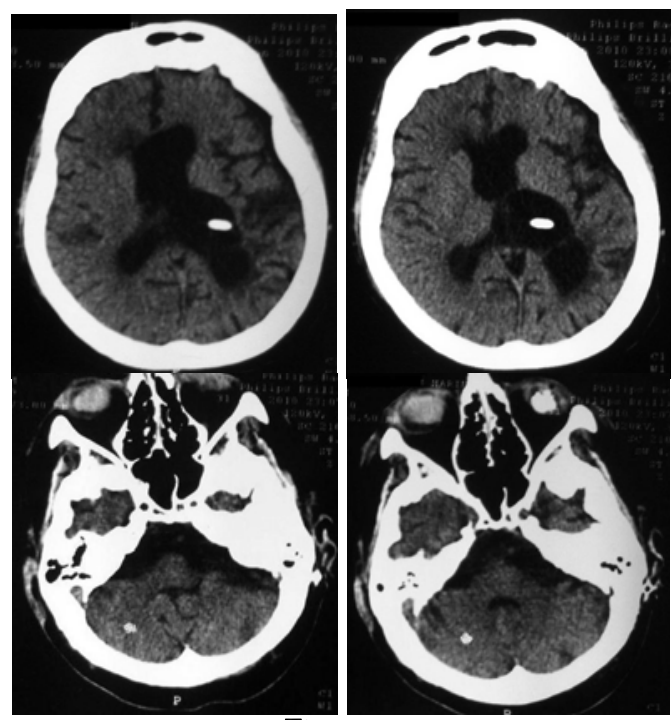

Figure 2

Cerebral CT. No tumor recurrence, left O porencephalic cyst, ventricular catheter, cerebellar hemisphere cavernoma with no signs of acute bleeding.
Seventeen years latter, in 2010, he was admitted with severe altered general state, comatouse state, with sudden onset 24 hours ago and acute respiratory failure. The CT showed no tumor recurrence within the ventricular system, left occipital porencephalic cyst, ventricular catheter in place and cerebellar hemisphere cavernoma with no signs of acute bleeding (figure 2).

The patient was diagnosed with ventriculoperitoneal shunt, left occipital porencephalic cyst, cortical atrophy, panhypopituitarism, optic atrophy, radioinduced unruptured cerebellar cavernoma, acute respiratory failure, pneumonia and right hemidiaphragm paralysis. Because the cavernoma was not ruptured, we chose conservative treatment. The outcome was slowly favorable, under hormonal therapy the patient regained consciousness, under antibiotic therapy pneumonia healed and respiratory failure improved.

\section{Case 3}

CC, M, 43 years old, was admitted for the first time, in 1992 with third ventricle tumor and secondary hydrocephalus. The patient underwent surgery, we performed a transcortical approach, total tumor resection and ventriculoperitoneal shunt. Histopathological exam was germinoma. Postoperative the patient underwent whole brain radiotherapy.

Twenty one years latter, in 2013, the patient came for a routine control. Cerebral CT and MRI revealed no tumor recurrence, ventricular catheter in place, no signs of active hydrocephalus and left frontal cavernoma without sings of acute 
bleeding (figure 3). The patient was diagnosed with ventriculoperitoneal shunt and radio-induced left frontal unruptured cavernoma. Due to the fact that the patient was asymptomatic and the cavernoma was unruptured we chose conservative treatment.

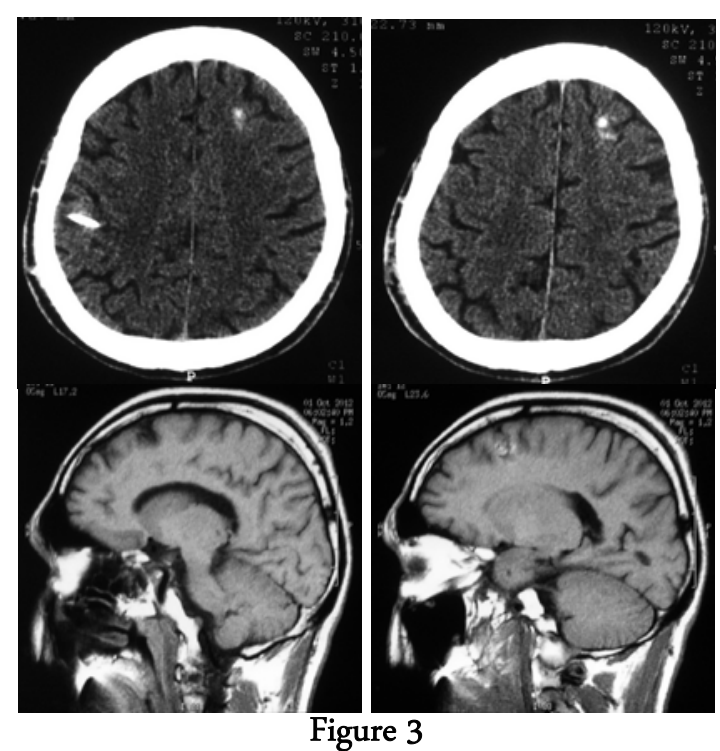

Cerebral CT \& MRI. No tumor recurrence, ventricular catheter, no hydrocephalus, left $\mathrm{F}$ cavernoma without sings of acute bleeding.

\section{Case 4}

PCM, M, 69 years old, was admitted in another department in 1982 with scalp tricophyton and underwent scalp irradiation.

Thirty years latter, in 2012, was admitted in our department with headache, ataxia and left parietal bulge. Cerebral CT showed a left parasagittal parietal cranioduro-cerebral tumor, well-defined, strongly enhancing contrast, with dural attachment, invading the SSS, with parietal bone lysis and extracranial extension (figure 4). The patient was diagnosed with left parietal cranio-duro-cerebral osteolytic tumor and partial optic atrophy. The patient underwent surgery and subtotal tumor resection was performed (the part infiltrating the SSS is left in place). Histopathological exam was anaplastic meningioma. Postoperative outcome was favorable, with no neurological deficits.

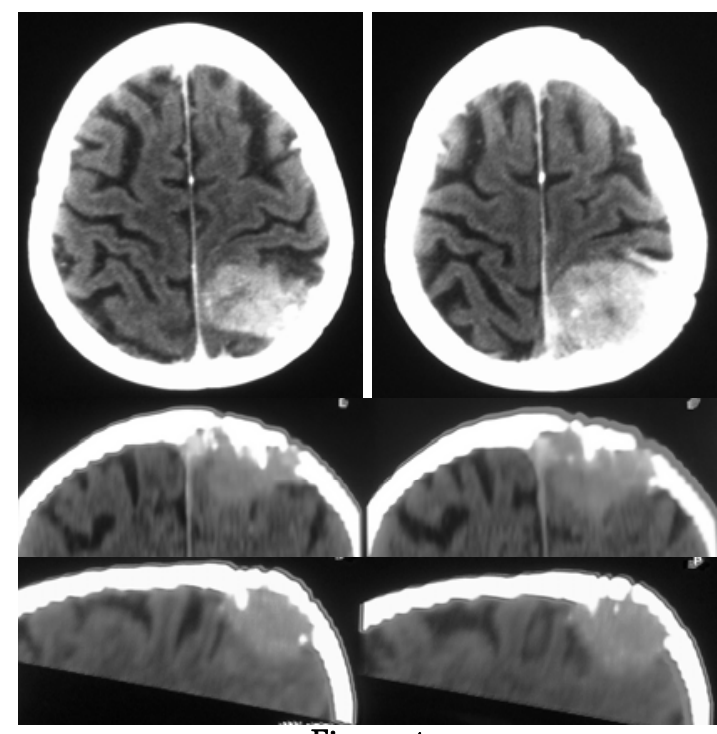

Figure 4

Cerebral CT. Left parasagittal P cranio-durocerebral tumor, well-defined, strongly enhancing contrast, with dural attachment, invading the SSS, with $\mathrm{P}$ bone lysis and extracranial extension.

\section{Discussions}

In our series of cases patients met Cahan's diagnostic criteria(2) for radioinduced brain lesions. Radiation side effects occur after a long period of time.(9;10) All four patients had radiotherapy, and after a sufficient long period of time, 13, 17, 21 and 30 years, another brain lesion with distinctive histopathology occurred. Radio-induced 
lesion occurred inside the radiation area. Taking into account the fact that these patients were irradiated long time ago, when focus radiation techniques were not in use, all patients had whole brain radiotherapy.

In our series of patients, fortunately, only benign pathology was noted, 2 patients were diagnosed with meningiomas and 2 with cavernomas. Meningiomas can grow very large, causing mass effect, progressively compressing surrounding structures and requiring surgery. Both patients with meningiomas had large tumors and none was asymptomatic at admission. They were operated with good outcome. In the other two patients with cavernomas, the vascular lesion was incidentally discovered, in one case at a routine control and in one case after altered general state that was not related to the cavernoma. In most of the situations cavernomas are asymptomatic, and require no surgery, unless they rupture or serial imaging show progression of the lesion. If any sign of progression is noted at followup, treatment options must be reconsidered.

Besides new tumor occurrence radiotherapy induced diffuse brain atrophy, leukoencephalopathy, optic atrophy and panhypopituitarism. Diffuse brain atrophy and leukoencephalopathy leads to neurocognitive impairment and progressive dementia.(12) Optic athophy causes visual loss. Panhypopituitarism is a condition of inadequate or absent production of anterior pituitary gland hormones. It is clinical manifest and requires synthetic hormonal replacement therapy with good outcome.

Because of the risk of tumor development any form of radiotherapy is contraindicated in children less 3 years old.(3)

Advances in radiotherapy, with focused, boost radiation techniques, that concentrates radiations into a target area, where the patients receives a high dose, and with protection of normal brain can diminish the risks of radio-induced brain lesion occurrence. Also fraction or hyperfraction of doses and the use of radioprotectants lower the risks for secondary tumor occurrence. Stereotactic radiosurgery seem to be a valid alternative to radiotherapy, with less complications. But, because stereotactic radiosurgery has less years of clinical use $(5 ; 6 ; 8)$ compare with conventional radiotherapy, further studies are needed to evaluate long-term complications following it. New devices, with low risk of irradiating normal brain are: linear accelerator Trilogy Varian, CyberKnife, robotic radiosurgery, CyberKnife VSI ${ }^{\circledR}, \quad$ Gamma Knife Perfexion ${ }^{\circledR}$, Novalis $\mathrm{Tx}^{\mathrm{TM}}$ radiosurgery platform.(1)

Thirty years ago the treatment for scalp tricophyton was scalp irradiation. Nowadays scalp irradiation is no longer used.

\section{Conclusions}

Radiotherapy can cause long-term complications and can induce development 
of new brain lesions into previous radiation area. Meningiomas and cavernomas may be radio-induced brain lesions and may occur following previous radiotherapy. Meningiomas can grow to large size, requiring surgery. Unruptured asymptomatic cavernomas can be left in place and patients are followed clinical and with serial imaging. Other findings after radiotherapy are diffuse brain atrophy, leukoencephalopathy, optic atrophy and panhypopituitarism.

\section{Abbreviations}

$C T$ - computer tomography

$M R I$ - magnetic resonance imaging

SSS - superior sagittal sinus

\section{Correspondence to:}

Aurelia Mihaela Sandu

Address: Fourth Department of Neurosurgery, Emergency Clinical Hospital Bagdasar-Arseni,

No. 10-12, Berceni Street, Sector 4, Bucharest; email: aurasandu@gmail.com; tel.0724.263.023

\section{References}

1. Adler JR Jr, Murphy MJ, Chang SD, Hancock SL. (1999). Image-guided robotic radiosurgery. Neurosurgery 44, 1299-1304.
2. Cahan WG, Woodard HQ, Higinbotham NL, Steward FW, Coley BL. (1948). Sarcoma arising in irradiated bone: report of 11 cases. Cancer 1, 3-29.

3. Greenberg MS. (2006). Handbook of neurosurgery. Thieme Medical Pulblishers.

4. Lacassagne A. (1933). Conditions dans lesquelles ont ete obtenus, cher le lapin, des cancers par actions des raisons x sur des foyers inflammatoires. C R Soc Biol 67, 244-251.

5. Leksell L. (1949). A stereotaxic apparatus for intracerebral surgery. Acta Chir Scand 99, 229-233.

6. Leksell L. (1951). The stereotaxic method and radiosurgery of the brain. Acta Chir Scand 102, 316-319. 7. Muracciole X, Cowen D, Regis J. (2004). Radiosurgery and brain radio-induced carcinogenesis: update. Neurochirurgie 50, 414-420.

8. Niranjan A, Kano H, Lunsford LD. (2013). Gamma Knife radiosurgery for brain vascular malformations. Basel (Switzerland), Karger.

9. Niranjan A, Konziolka D, Lundsford D. (2009). Neoplastic transformation after radiosurgery or radiotherapy: risk and realities. Otolaryngol Clin North Am 22, 717-729.

10. Salvati M, DElia A, Melone GA, Brogna C, Frati A, Raco A, Delfini R. (2012). Radio-induced gliomas: 20year experience and critical review of the pathology. J Neurooncol 89, 169-177.

11. Salvati M, Frati A, Russo N, Caroli E, Polli FM, Minniti G, Delfini R. (2003). Radio-induced gliomas: report of 10 cases and review of the literature. Surg Neurol 60, 67.

12. Tallet AV, Azria D, Barlesi F, Spano JP, Carpentier AF, Goncalves A, Metellus P. (2012). Neurocognitive function impairment after whole brain radiotherapy for brain metastases: actual assessment. Radiat Oncol 7, 77. 